\title{
Simultaneous optimisation of tie switches placement and reserve capacity margin of sub-transmission substations considering the conflict between short-term and long- term plannings
}

\author{
Milad Hoseinpour ${ }^{凶}$, Mahmoud-Reza Haghifam ${ }^{1}$, \\ Mansoreh Zangiabadi ${ }^{2}$, Sayed Majid Miri Larimi ${ }^{1}$ \\ ${ }^{1}$ Department of Electrical and Computer Engineering, Tarbiat Modares University, Tehran, Iran \\ ${ }^{2}$ Department of Electrical and Computer Engineering, Newcastle University, Newcastle upon \\ Tyne, UK \\ $凶$ E-mail: m.hoseinpour@modares.ac.ir
}

\begin{abstract}
Outage management has a crucial role in improving reliability level of distribution networks, and it is profoundly dependent on the reserve capacity margin of sub-transmission (ST) substations and load transferring capability between them. These factors enable distribution system operator (DSOs) to restore interrupted load points in out-of-service areas. In this study, the connection of reserve capacity in ST substations and load transferring capability, made by tie switches, is studied. The mentioned correlation is addressed via costumer interruption cost derived from outage management in primary distribution networks. Then, a mathematical model is developed in which optimal reserve capacity of ST substations and optimal placement of tie switches are determined simultaneously. The resulted optimisation model is formulated as a mixed-integer non-linear problem, and the genetic algorithm is used to solve the problem. Finally, the effectiveness of the proposed model is evaluated in a test distribution network.
\end{abstract}

\section{Nomenclature \\ Indices \\ $i$ index of ST substations \\ $j$ index of candidate tie points \\ $b$ index of network branches \\ $\sigma$ index of interrupted load points \\ $n$ index of network nodes \\ $S$ Index of feeder sections}

\section{Sets}

$\Omega$ set of ST substations

$\Lambda$ set of network branches

$N$ set of network nodes

$K$ set of control variables

$N d l p, b$ set of interrupted load points due to fault in branch $b$

\section{Parameters}

$n_{\mathrm{ST}}$ number of ST substations

$N_{\mathrm{T}}$ number of candidate tie points

$N_{\mathrm{b}}$ number of network branches

ICT installation cost of a tie switch (\$/switch)

TCC tie-line creation cost $(\$ / \mathrm{km})$

$l_{j}$ length of the tie line in candidate tie point $j(\mathrm{~km})$

$\lambda_{\mathrm{b}}$ failure rate of branch $b$ (fail/year)

$r_{\mathrm{b}}$ repair time of branch $b(\mathrm{~h})$

$\Gamma_{\sigma, \mathrm{b}}$ interruption cost in load point $\sigma$ during fault in branch $b$

$(\$ / \mathrm{kW})$

$t$ study period (year)

int $_{\mathrm{rt}}$ interest rate $d_{\sigma, \mathrm{b}}$ demand of load point $\sigma$ which could not be restored due to failure in branch $b(\mathrm{~kW})$

$d_{i}$ load demand of main transformer in ST substation $i$ (MVA)

\author{
Variables \\ $\gamma_{i}$ utilisation factor of ST substation $i$ \\ $\psi_{j}$ presence or absence of tie switch in candidate location $j$ \\ $C_{\mathrm{ST}, i}$ total capacity of ST substation $i$ (MVA) \\ $v_{\text {cap }}^{i}$ capacity of virtual transformer in ST substation $i$ (MVA) \\ $v_{\mathrm{IC}}^{i}$ installation cost of virtual transformer in ST substation $i(\$)$ \\ MCC manoeuvre creation cost (\$) \\ $\mathrm{CIC}_{b}$ customer interruption cost because of failure in branch $b(\$)$ \\ $\tau_{\mathrm{L}, b}^{i}$ transferred load to ST substation $i$ because of failure in branch \\ $b(\mathrm{~kW})$
}

\section{Introduction}

Today distribution companies (discos) are in a time of changes and challenges. The main changes come with distribution networks liberalisation, modernisation, automation, and more efficient utilisation of network assets. On the other side, the most important challenge is the increasingly demand of customers for reliability regard to increasingly growth of critical loads such as digital loads. So, discos should adapt for the changes in such a way that is in line with challenges and manage their assets inclined to their objective, on top of them network reliability. However, there is a conflict between improving the service reliability and controlling of costs. In this emerging environment of changes and challenges, asset management plays a key role. Asset management signifies strategic planning, maintenance, utilisation, and operation of a physical resource. In addition, possible time scopes of it contain 
real-time, short-term, mid-term and long-term. From a practical prospective, there are interactions between aforementioned time scopes. Furthermore, it has been known that the coordination of asset management strategies in different time scopes has a significant impact in decision-making process of a disco. An overall view into the prominent interactions in asset management strategies of distribution networks is given in Fig. 1.

High proportions of customer's interruptions are caused by failures in primary distribution networks [1]. Therefore, it is rational that special attention is paid to improve the reliability of distribution networks in asset management strategies [2]. One of the efficient methods for relieving customer's interruption duration, and also improving reliability, is to provide reserve capacity in ST substations and feeders [3,4]. With this additional capacity in ST substations and by using the capability of the normally closed and opened switches in primary distribution networks, the DSO can restore out-of-service areas from other routes [5].

The capability of tie switches for transferring interrupted load points to adjacent ST substations is highly dependent on available reserve capacity. However, in the literatures high share of works concern about placement of tie switches and switching devices in reliability optimisation problems regardless of the correlation of tie switches and the reserve capacity of ST substations. In [6], to improve the system reliability with distributed generation under fault conditions, tie-lines placement schemes through graph-based algorithms have been proposed. A micro-genetic algorithm (GA), in conjunction with the fuzzy logic, for modelling expert knowledge has been proposed as a solution for optimal placement of sectionalisers and tie switches in [7]. An ant colony optimisation method for placement of sectionalising switches in the presence of distributed generation sources by using a fuzzy multi-objective approach has been illustrated in [8]. Particle swarm optimisation algorithm was developed to determine optimum number and locations of sectionalisers and breakers [9]. In addition, optimum number of switches in a distribution network

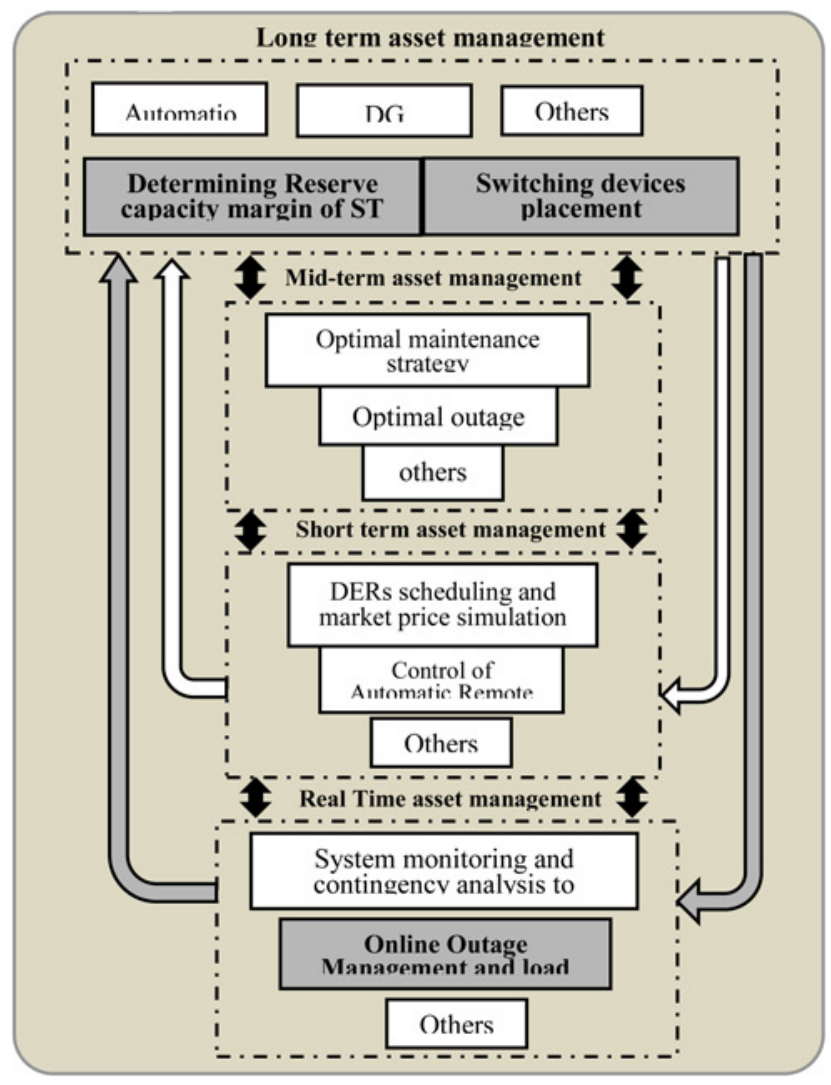

Fig. 1 Prominent interactions in asset management strategies of the distribution networks was obtained by cost analysis on a system, and optimum locations of switches were found by simulated annealing algorithm [10]. As mentioned earlier, studies which relate to reserve capacity margin of ST substations are rare regardless of vast studies in switching devices and load transferring capability. For example, the capacity of an ST substation was empirically analysed based on single-contingency policy (SCP), which assumes that adjacent ST substations should support one another when one of them is unavailable [11-13]. In addition to SCP for the determination of reserve capacity in ST substations, the capacity/load ratio is a popular and useful index in ST substations capacity planning $[14,15]$. Despite low efficiency and lack of accurate mathematical validation, this approach is practical and has been widely used by engineers. The aforementioned studies do not give the optimal solution because the supply capability of distribution networks could not be efficiently utilised. With regard to growing tendency and satisfying consequences derived from implementation of asset management activities in discos, researchers implement more efficient and accurate approaches for using the supply capabilities of distribution networks in which multiple ST substations are interconnected with normally open switches $[16,17]$. Plus, in the previous studies, real-time, short-term, mid-term, and long-term asset management activities were performed independently, whereas coordinated planning in distribution networks reduces overall cost of discos [3]. In this regard, highlighted boxes in Fig. 1 indicate the interactions that are considered in the present study. Owing to these highlighted boxes, the main contribution of this paper is the coordination between long-term decisions, which are the determination of ST substations reserve capacity and tie switches placement, and real-time decisions, which is outage management using tie switches.

The rest of this paper is organised as follows: Section 2 presents the proposed model of the problem. Section 3 discusses formulations and outage management process. Section 4 provides the codification of the problem. Section 5 investigates the results of simulation. Finally in Section 6, the conclusion of this paper is presented.

\section{Description of the proposed model}

As mentioned earlier, we propose a model that considers the interaction between real-time and long-term asset management strategies of a disco. In this work, the reserve capacity is modelled as a small individual virtual transformer in an ST substation apart from the capacity of the main transformer which was obtained from the forecasted demand. As discussed in the previous section, the reserve capacity of ST substations depends on the number and location of tie switches. The correlation between the reserve capacity and tie switches is investigated in fault condition through outage management strategies, and finally the optimal decisions about the reserve capacity and tie switches placement are made. The conceptual framework of the proposed model is illustrated for a distribution network with two feeders and $m$ candidate tie switches in Fig. 2.

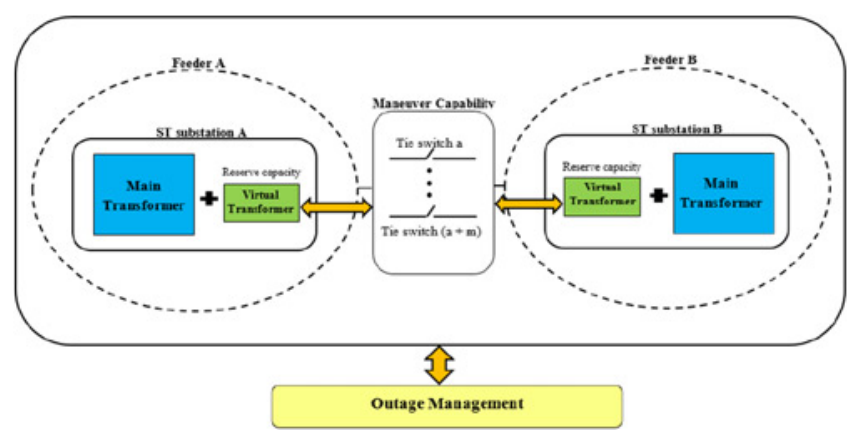

Fig. 2 Conceptual framework of the proposed model 


\section{Problem formulation}

The decision-making variables in this paper are considered as an $\kappa: 1 \times\left(n_{\mathrm{ST}}+N\right)$ array, where $n_{\mathrm{ST}}$ and $N_{T}$ represent the number of the ST substations and candidates for tie switches placement, respectively. The set of decision-making variables of this paper include continuous variables $\gamma_{i}: \kappa_{i}, 1 \leq i \leq n_{\mathrm{ST}}$ and binary variables $\quad \psi_{j}: \kappa_{j},\left(n_{\mathrm{ST}}+1\right) \leq j \leq\left(n_{\mathrm{ST}}+N\right) . \quad \psi_{j}$ denotes the presence or absence (1: presence and 0 : absence) of tie switch in candidate location $j$. Also $\gamma_{i}$ represents the utilisation factor of ST substation $i$ with respect to the utilisation factor, the total capacity of ST substation $i$, which consists of the forecasted load demand of ST substation $i$ and the capacity of virtual transformer in it, can be derived as below:

$$
C_{\mathrm{ST}, i}=\frac{d_{i}}{\gamma_{i}}, \quad 1 \leq i \leq n_{\mathrm{ST}}
$$

Hence the reserve capacity or the capacity of virtual transformer can be computed based on (2)

$$
v_{\text {cap }}^{i}=d_{i} \frac{1-\gamma_{i}}{\gamma_{i}}, \quad 1 \leq i \leq n_{\mathrm{ST}}
$$

The mathematical model resulted from these assumptions makes a mixed-integer non-linear programming (MINLP) problem. In what presented in the following, formulations are described in detail.

\subsection{Investment cost}

The investment cost consists of two parts: the first part relates to the installation cost of the virtual transformer with capacity of $v_{\text {cap }}^{i}$ and can be formulated as

$$
v_{\mathrm{IC}}^{i}=\left\{\begin{array}{lll}
V_{\mathrm{IC}, 1} & \text { if, } & v_{\text {cap }, 1} \leq v_{\text {cap }}^{i}<v_{\text {cap }, 2} \\
V_{\mathrm{IC}, 2} & \text { if, } & v_{\text {cap }, 2} \leq v_{\text {cap }}^{i}<v_{\text {cap }, 3} \\
\cdot & \cdot & \\
\cdot & \cdot & \\
V_{\mathrm{IC}, m-1} & \text { if, } & v_{\text {cap }, m-1} \leq v_{\text {cap }}^{i} \leq v_{\text {cap }, m}
\end{array}\right.
$$

The second part of the investment cost relates to manoeuvre creation cost (MCC) which can be formulated as

$$
\mathrm{MCC}=\sum_{j=1}^{N} \psi_{j}\left(\mathrm{ICT}+1_{j} \mathrm{TCC}\right)
$$

\subsection{Reliability cost}

For computing customer interruption cost (CIC) after fault creation in each branch, interrupted load points are found, then load restoration is done. In load restoration with considering interrupted load points and the set of available tie points, all network configurations which can restore these load points are examined. If sum of the interrupted load points connected to ST substation is higher than $v_{\text {cap }}^{i}$, a load point is disconnected from end of the interrupted feeder. This procedure is repeated until non-delivered power is equal or lower than the capacity of virtual transformer and then forward-backward sweep load flow is implemented to check the voltage and current constraints in the new topology. If there is no constraints violation in the new topology, the loads are restored; otherwise, a load point is disconnected from end of interrupted feeder and added to CIC. Customer in this section, the proposed model for coordinate determining of the optimal reserve capacity of ST substations and the placement of tie switches is that the switching time is negligible

$$
\mathrm{CIC}_{b}=\left(\lambda_{b} \sum_{\sigma=1}^{N d l p, b} d_{\sigma, b} \Gamma_{\sigma, b}\right)\left(1-\text { int }_{\mathrm{rt}}\right)^{t-1}
$$

\subsection{Objective function}

The objective function of the proposed MINLP problem is to minimise the total cost from the viewpoint of a distribution company; therefore, the resulting optimisation problem is

$$
\min _{\kappa}\left\{\sum_{i=1}^{n_{\mathrm{ST}}} v_{\mathrm{IC}}^{i}+\sum_{b=1}^{N_{b}} \mathrm{CIC}_{b}+\sum_{j=1}^{N} \psi_{j}\left(\mathrm{ICT}+1_{j} \mathrm{TCC}\right)\right\}
$$

subject to:

(i) Utilisation factor

$$
0 \leq \gamma_{i} \leq 1, \quad \forall i \in \Omega
$$

(ii) Load transferring constraint during load restoration because of fault in branch $b$

$$
\tau_{\mathrm{L}, b}^{i} \leq v_{\text {cap }}^{i}, \quad \forall i \in \Omega
$$

(iii) Voltage and current constraints after load restoration

$$
\begin{gathered}
V_{\min } \leq V_{n} \leq V_{\max }, \quad \forall n \in N \\
\left|I_{b}\right| \leq I_{\max }, \quad \forall b \in \Lambda
\end{gathered}
$$

\section{Codification}

The proposed optimisation problem is an MINLP problem which is solved through GA. The coding strategy of this paper is shown in, in which the proposed chromosome is composed of two strings. The first and second strings represent the utilisation factor of each ST substation and tie points allocation, respectively. In the string which represents the utilisation factors of ST substations, each gene contains a value between 0 and 1 , and in the string representing tie points allocation each gene contains a binary value. This binary coding characterises the candidate locations for tie switches installation in the network. The values 1 and 0 in gene $N$ represent 'existing' and 'not existing' of a tie point in the candidate location $N$ (Fig. 3).

\section{Numerical analysis}

In this section, the proposed model for coordinate determining of the optimal reserve capacity of ST substations and the placement of tie switches is investigated. The proposed model is applied to a

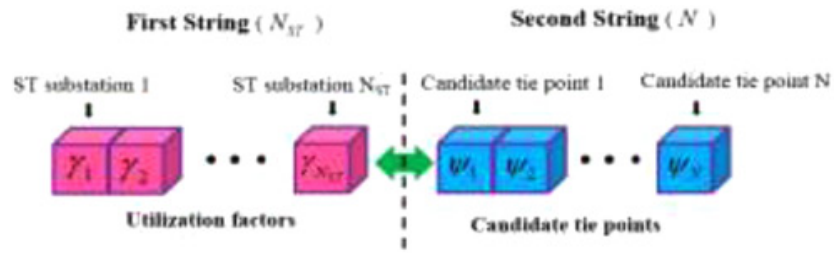

Fig. 3 Implemented codification scheme 
typical $20 \mathrm{kV}$ urban distribution network (Fig. 4). There are five 63/ $20 \mathrm{kV}$ ST substations and 82 medium-voltage/low-voltage distribution substations in the network. Service area of each ST substation is separated by automatic normally open switches. In addition, there are automatic normally close switches in each section between two distribution substations, and in order to avoid complication of the network diagram, these switches are not depicted in Fig. 4. In this test case, the designer engineer considers six candidate tie points. Also, the input parameters are shown in Table 1, and the interruption cost for all load points including residential, commercial, and industrial are calculated based on customer damage function in [18].

Case 1: no tie point is available in the network for load restoration. So, as we expected, the utilisation factors of the ST substations derived from the model are equally 1 . It means that there would be no reserve capacity in the network to restore the interrupted load points in emergency conditions.

Case 2: It is dedicated to the main idea of this paper which is about coordinated optimisation of tie points placements and the reserve margin of ST substations. In Table 2, the optimal tie points for the proposed network, with regard to the interaction of the reserve margin and tie points, are found, which are T1, T3, T4, and T5. Among the ST substations, ST substation 4 has the maximum number of tie points and the maximum reserve capacity interpreting the significant CIC of feeders connected to the ST

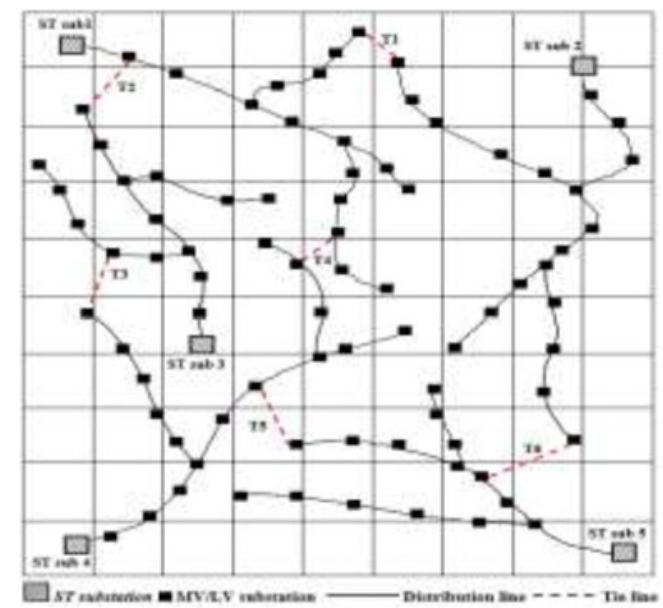

Fig. 4 Test case

Table 1 Input parameters

\begin{tabular}{lcc}
\hline Technical and economical information & & \\
\hline Parameter & Unit & Value \\
\hline$t$ & year & 10 \\
$V_{\text {IC }}$ & $\$, \mathrm{MVA}$ & 20,000 \\
installation cost of a tie switch & $\$$, switch & 5000 \\
tie-line creation cost & $\$, \mathrm{~km}$ & 19,000 \\
\hline
\end{tabular}

Table 2 Results of case 2

\begin{tabular}{lccccc}
\hline $\begin{array}{l}\text { ST } \\
\text { sub }\end{array}$ & $\begin{array}{c}\text { Demand, } \\
\text { MVA }\end{array}$ & $\begin{array}{c}\text { Utilisation } \\
\text { factor, opc. }\end{array}$ & $\begin{array}{c}\text { Total } \\
\text { capacity, } \\
\text { MVA }\end{array}$ & $\begin{array}{c}\text { Reserve, } \\
\text { MVA }\end{array}$ & $\begin{array}{c}\text { Linked } \\
\text { tie points }\end{array}$ \\
\hline 1 & 3.25 & 0.62 & 5.24 & 1.99 & T3, T4 \\
2 & 3.57 & 1 & 3.57 & 0 & TI \\
3 & 2.95 & 0.81 & 3.64 & 0.69 & T3 \\
4 & 3.35 & 0.62 & 5.4 & 2.05 & T3, T4, \\
& 3.85 & 0.86 & 4.47 & 0.62 & T5 \\
\hline
\end{tabular}

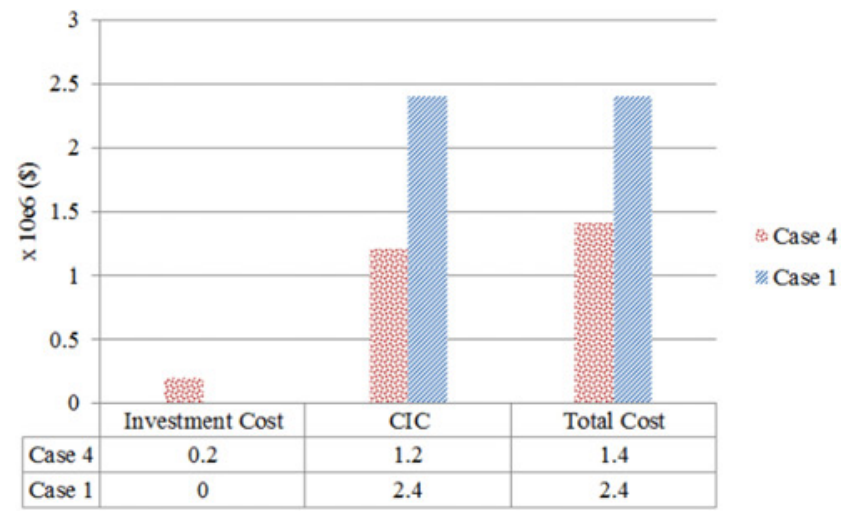

Fig. 5 Comparison between cases 1 and 2

substation 4 through the tie points. As shown in Table 2, no reserve capacity is allocated to ST substation 2; however, this ST substation is connected through T1 to the ST substation 1. This observation shows that if any fault occurs in feeder 1 , the interrupted load points could not transfer to ST substation 2, and the interrupted load points are restored through T4 from the ST substation 4 . Then, the motivation of the existence of $\mathrm{T} 1$ is only for restoration of the interrupted load points in feeder 2 under fault condition; in other words, ST substation 1 is a backup for ST substation 2, whereas the ST substation 2 is not a backup for it.

A comparison is made between the case 2 and case 1 (Fig. 5), which is assumed there is no tie point in the network. In case 2 leading to optimal solution, a remarkable plunge is observed in CIC, and therefore in the total cost, because of the tie points which are placed with regard to the connection of load transferring capability and the reserve capacity margin of ST substation.

\section{Conclusion}

This paper focuses on the correlation of the reserve capacity margin of ST substations and tie points placement, and based on this correlation it proposes a model for coordinated optimisation of the reserve capacity in ST substations and network tie points. The high value of CIC in the first case reveals the adverse consequence of the lack of tie points in the network. It is shown that a small investment in tie points creation and the reserve capacity makes a decisive plunge in $\mathrm{CIC}$, and the optimal solution is obtained by trade-off between CIC and investment cost.

\section{References}

1 Brown, R.E.: 'Electric power distribution reliability' (CRC Press, 2008)

2 Celli, G., Pilo, F. 'Optimal sectionalizing switches allocation in distribution networks', IEEE Trans. Power Deliv., 1999, 14, (3), pp. 1167-1172

3 Salehi, J., Haghifam, M.R.: 'Determining the optimal reserve capacity margin of sub-transmission (ST) substations using genetic algorithm', Int. Trans. Electr. Energy Syst., 2014, 24, (4), pp. 492-503

4 Lakervi, E., Holmes, E.J.: 'Electricity distribution network design' (IET, 1995)

5 Haghifam, M.R.: 'Optimal allocation of tie points in radial distribution systems using a genetic algorithm', Eur. Trans. Electr. Power, 2004, 14, (2), pp. 85-96

6 Mao, Y., Miu, K.N.: 'Switch placement to improve system reliability for radial distribution systems with distributed generation', IEEE Trans. Power Syst., 2003, 18, (4), pp. 1346-1352

7 do Nascimento Alves, H.: 'A hybrid application based on expert knowledge for solving the switches placement problem in distribution networks', J. Control Autom. Electr. Syst., 2014, 25, (2), pp. 252-261

8 Moradi, A., Fotuhi-Firuzabad, M.: 'Optimal switch placement in distribution systems using trinary particle swarm optimization algorithm', IEEE Trans. Power Deliv., 2008, 23, (1), pp. 271-279

9 Falaghi, H., Haghifam, M.-R., Singh, C.: 'Ant colony optimization-based method for placement of sectionalizing switches in distribution networks using a fuzzy multiobjective approach', IEEE Trans. Power Deliv., 2009, 24, (1), pp. 268-276

10 Billinton, R., Jonnavithula, S.: 'Optimal switching device placement in radial distribution systems', IEEE Trans. Power Deliv., 1996, 11, (3), pp. 1646-1651 
11 Luo, F., Wang, C., Xiao, J.,, et al.: 'Rapid evaluation method for power supply capability of urban distribution system based on $N-1$ contingency analysis of main-transformers', Int. J. Electr. Power Energy Syst., 2010, 32, (10), pp. 1063-1068

12 Wang, J., Carvalho, P., Kirtley, J.: 'Emergency reconfiguration and distribution system planning under the single-contingency policy'. 2012 IEEE PES Innovative Smart Grid Technologies (ISGT), 2012

13 Xiao, J., Li, X., Gu, W.,, et al.: 'Model of distribution system total supply capability considering feeder and substation transformer contingencies', Int. J. Electr. Power Energy Syst., 2015, 65, pp. 419-424

14 Peng, W., Pan, J.: 'Rural electrification in China: history and institution', China World Econ., 2006, 14, (1), pp. 71-84
15 Liu, S.-G., Cui, Y.-Y., Zhang, Z.-P.: 'Research on rational capacity-load ratio of urban electric network'. 2010 China Int. Conf. Electricity Distribution (CICED), 2010

16 Xiao, J., Li, F., Gu, W.,, et al.: 'Total supply capability and its extended indices for distribution systems: definition, model calculation and applications', IET Gener. Transm. Distrib., 2011, 5, (8), pp. 869-876

17 Xiao, J., Li, F., Gu, W.,, et al.: 'Total supply capability and its extended indices for distribution systems: definition, model calculation and applications', IEEE Trans. Syst., 2009, 24, (2), pp. 1019-1028

18 Chowdhury, A., Koval, D.: 'Power distribution system reliability: practical methods and applications' (John Wiley \& Sons, 2011) 